\title{
Herbal Formula Gegen-Qinlian Decoction for Type 2 Diabetes Mellitus: A Meta-Analysis of Randomized Controlled Trials
}

\author{
Lin Ren, ${ }^{1}$ Yanxia Cheng, ${ }^{1}$ and Feng Qin (iD) ${ }^{2}$ \\ ${ }^{1}$ Department of Pharmacy, West China Hospital, Sichuan University, Chengdu 610041, China \\ ${ }^{2}$ Andrology Laboratory, West China Hospital, Sichuan University, Chengdu 610041, China \\ Correspondence should be addressed to Feng Qin; 174299227@qq.com
}

Received 29 May 2020; Revised 29 September 2020; Accepted 7 October 2020; Published 21 October 2020

Academic Editor: Chan-Yen Kuo

Copyright $(2020$ Lin Ren et al. This is an open access article distributed under the Creative Commons Attribution License, which permits unrestricted use, distribution, and reproduction in any medium, provided the original work is properly cited.

Background. Herbal formula Gegen-Qinlian Decoction (GQD) has been widely used in China for the treatment of type 2 diabetes mellitus (T2DM), but its efficacy and safety are unclear. Method. The studies were identified from the PubMed, EMBASE, Cochrane Library, China National Knowledge Infrastructure database, Wanfang database, and VIP database using the keywords "Gegenqinlian” or "Gegen-Qinlian” or "Gegen-Qin-Lian” or "Ge Gen Qin Lian.” Relevant studies were selected according to predefined inclusion and exclusion criteria. Study selection, data extraction, and validation were carried out by, at least, two reviewers with disagreements being settled by discussion. Results. After literature search, a total of 26 randomized controlled trials were included with a total of 2553 patients. There was evidence that compared with metformin, the combination of GQD and metformin significantly reduced the fasting plasma glucose levels (MD -1.79, 95\% CI $(-2.31,-1.27), p<0.00001)$; 2 -hour postprandial plasma glucose levels (MD $-1.72,95 \%$ CI $(-2.12,-1.31), p<0.00001)$; and glycosylated hemoglobin levels (MD $-1.26,95 \%$ CI $(-1.80,-0.72), p<0.00001)$, and no serious side effects were identified. Conclusion. These data suggest that GQD may be an effective herbal formula in treating T2DM without serious side effects. The addition of GQD also enhances the hypoglycemic effects of metformin. However, the evidence remains weak due to methodological flaws, which may amplify the therapeutic benefit of GQD.

\section{Introduction}

Diabetes is a common and frequently occurring disease, seriously harming the human health. Diabetes is a group of clinical syndromes characterized by hyperglycemia. The main types are type 1 diabetes, type 2 diabetes, and gestational diabetes, and type 2 diabetes is the most prevalent form [1]. Type 2 diabetes mellitus (T2DM) is a metabolic disease characterized by chronic inflammation, insulin resistance, and islet cells damagement [2]. Long-term chronic hyperglycemia may cause microvascular disease, neuropathy, retinopathy, diabetic foot, diabetic nephropathy, and other diseases $[3,4]$. Metformin is currently used as the firstline choice for the pharmacologic treatment of T2DM, but $20-30 \%$ of people develop gastrointestinal side effects, and $5 \%$ are unable to tolerate metformin due to these side effects [5]. Therefore, it has been gaining significant importance to search better agents worldwide from herbs or natural products in the recent years.

Gegen-Qinlian Decoction (GQD) is a classical herbal formula, which was firstly recorded in Shang-Han-Lun (Treatise on Febrile Diseases) of the Han Dynasty (202 BC$220 \mathrm{AD})$. GQD is widely used to treat diarrhea and diabetes in Chinese clinical practice [6-8]. It contains the following 4 herbs: Gegen (Puerariae Lobatae Radix), Huanglian (Coptidis Rhizoma), Huangqin (Scutellariae Radix), and Gancao (Glycyrrhizae Radix et Rhizoma). It had been reported that GQD could significantly decrease fasting blood glucose, glycosylated serum protein, glycosylated hemoglobin, and fasting serum insulin and promote myocardial glycolysis in diabetic rats $[9,10]$. Isoflavonoids $\left(3^{\prime}\right.$-hydroxy puerarin, puerarin, daidzin, daidzein, genistin, and genistein), flavonoids (baicalin, baicalein, wogonoside, wogonin, liquiritin, and liquiritigenin), alkaloids (berberine, jatrorrhizine, 
palmatine, and coptisine), and glycyrrhetic acid have been identified within the preparation [11-13], and these components are correlated to the antidiabetic, antioxidant, and immunoregulative effects [14-17]. Notably, the administration of GQD has also yielded a potential hypoglycemic effect associated with multitarget therapy.

The greatest hindrance for the acceptance of herbal formula in the world is the scientific evaluation. Despite the extensive use of GQD in China, most of the evidence about GQD are anecdotal and have not been properly studied with scientifically rigorous trials, especially on human subjects.

The primary objective of this study is to determine the effectiveness and safety of GQD in the treatment of T2DM utilizing a meta-analysis approach. At the same time, we hope to find out the deficiencies in the use of GQD and find the direction for future research.

\section{Methods}

2.1. Literature Search. The study was registered in the PROSPERO database with ID CRD42020209404. No language restriction was imposed on the literature search. The literature search was performed using a combination of the term T2DM and the following keywords: Gegenqinlian or Gegen-Qinlian or Gegen-Qin-Lian or Ge-Gen-Qin-Lian. The databases that were searched included PUBMED (1966 to December 2019), EMBASE (1980 to December 2019), CNKI database (1994 to December 2019), Wanfang Data (1989 to December 2019), VIP Information (1990 to December 2019), and the Cochrane Library (Issue 12, 2019). A secondary search was also conducted by searching reference lists from primary studies, as well as former reviews.

2.2. Inclusion and Exclusion Criteria. Two reviewers (L.R. and F.Q.) independently decided which trials fit the inclusion and exclusion criteria for this study. Studies were eligible for inclusion if they met all of the following criteria: (1) study design: all participants were randomly allocated to an experimental group and a control group, and both parallel and crossover studies were eligible. (2) Target population: all participants were aged 18 years and above. (3) Diagnostic criteria: all participants were diagnosed as having T2DM according to the China guideline for T2DM [18] or WHO diagnostic criteria for T2DM [19]. (4) Comparison: studies had to compare GQD with metformin. (5) Outcome: studies have used data based on fasting plasma glucose (FPG) or 2 Hours Postprandial Plasma Glucose (2hPPG), or glycosylated hemoglobin (HbAlc) as primary outcomes.

Studies were eligible for exclusion if they met any of the following criteria: (1) case reports, animal studies, nonclinical studies, and reviews. (2) Unverified randomized controlled trial (RCT). (3) No appropriate experimental group or control group. (4) Duplicate publications.

2.3. Data Extraction. Two independent reviewers (L.R. and F.Q.) extracted data from the relevant studies using a standard data collection form in order to avoid bias in the process. All data were assessed for internal consistency, and inconsistencies were discussed by the three reviewers (L.R., F.Q., and Y.X.C.) when necessary. The following information was obtained: (1) the name of the author, (2) the date of publication, (3) the duration of the treatment, (4) the diagnostic criteria, (5) the age and gender of the participants, (6) the course of the disease, (7) the number of participants, (8) the intervention drugs (dosage and preparations), (9) primary outcomes, and (10) side effects. When necessary, additional information was collected through collaboration with the authors.

The quality assessment of the RCTs was also determined independently by two reviewers (L.R. and F.Q.) using the Cochrane risk of bias tool. Discrepancies were discussed by the three reviewers (L.R., F.Q., and Y.X.C.). According to our previous method [20], the study was designed to assess (1) random sequence generation, (2) allocation concealment, (3) blinding of participants and personnel, (4) blinding of outcome assessment, (5) reporting of dropout or withdrawal, (6) selective outcome reporting, and (7) other potential bias. Each item was rated as low risk of bias, high risk of bias, or unclear risk of bias.

2.4. Statistical Analysis. The trials that were included in the present study performed the following comparisons: GQD versus metformin; GQD plus metformin versus metformin. Meta-analysis was performed using Review Manager Software (version 5.4, Cochrane Collaboration and Updated Software). Mean difference (MD) with $95 \%$ confidence intervals (CI) was calculated since the data units were consistent. A fixed-effect model can be more appropriate when there is statistical homogeneity $\left(p>0.1\right.$ or an $I^{2}$ statistic $<50 \%$ ) among the studies, and random-effect model need to be pursued when statistical heterogeneity $(p<0.1$ or an $I^{2}$ statistic $>50 \%$ ) exists in the trials. Funnel plot analysis and Egger's test were used to detect publication bias.

\section{Results}

3.1. Literature Search. An overview of the study selection process is summarized in Figure 1. The electronic search strategy identified 533 potentially relevant studies after accurate evaluation of the abstracts. In cases of disagreement as to whether an article was relevant, the full original article was retrieved for assessment. Of the 533 resulting studies, there were 243 duplicates, 81 reviews, 74 animal experiments, 27 nonclinical trials, and 14 not related to T2DM. After reading the full texts of the remaining 94 studies, 24 control groups were not metformin or unknown, 27 treatment groups contained other herbal medicine, 14 studies failed to provide useful data, 3 studies were not designed properly, and the remaining 26 studies were included in the meta-analysis [21-46].

3.2. Methodological Quality of Studies Included. According to the Cochrane risk of bias assessment tool, the methodologic quality item for all included studies is described in Figure 2. In general, the methodological quality of the 26 studies was low. Of the 26 studies, 21 studies reported 


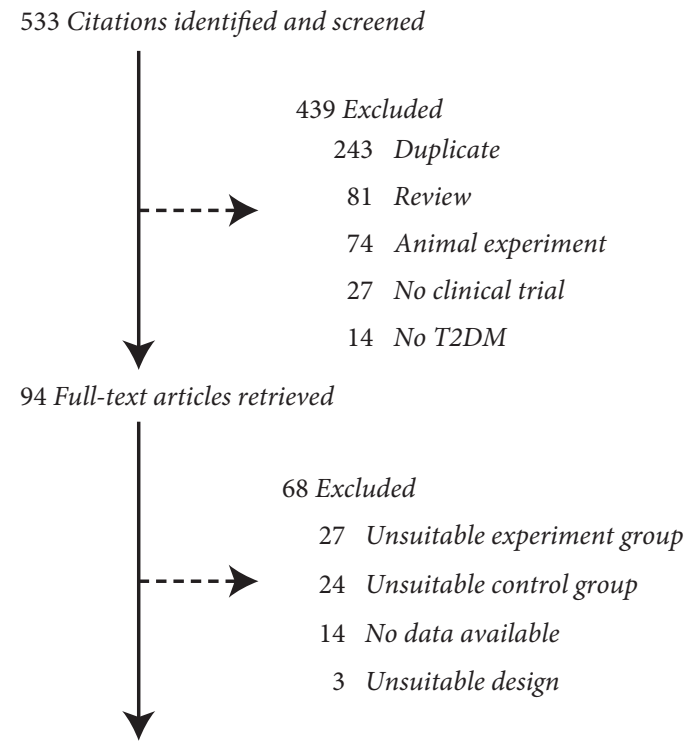

26 Articles finally enrolled in analysis

Figure 1: Study selection process for the meta-analysis.

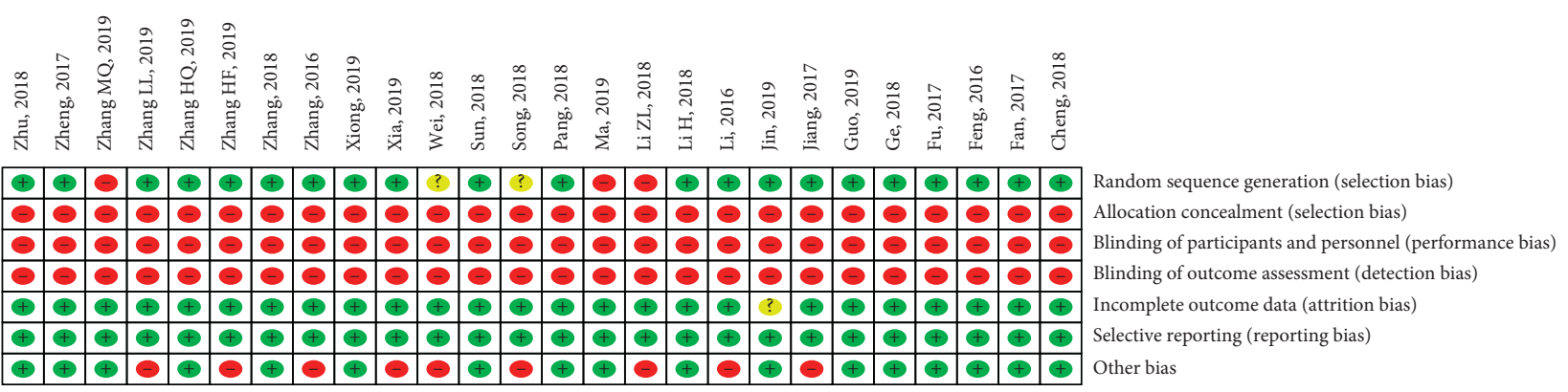

FIGURE 2: Methodological quality assessment of the risk of bias. Low risk of bias; unclear risk of bias; and high risk of bias.

random sequence generation, 3 studies have high risk on generating random sequence $[29,41,46]$, and the other 2 studies did not describe random sequence generation $[28,31]$. All the RCTs failed to describe the allocation concealment, blinding of participants and personnel, and blinding of outcome assessors in detail. There was no missing data in all the studies except the study of Jin [43], which did not mention the number of people who completed. The dose of metformin in 9 studies was not fixed, which was judged as high risks of other potential bias [26-29, 31, 32, 37, 38, 44].

3.3. Study Characteristics. Twenty-six studies, with 2553 participants, were included in the meta-analysis. All of the studies were performed in China. The studies were conducted between June 2010 and December 2019, and the dates of publication ranged from 2012 to 2019. The duration of intervention ranged from 2 to 24 weeks. Twenty-six trials reported data for FPG $(n=2553), 22$ for $2 \mathrm{hPPG}(n=2261)$, and 20 for HbAlc $(n=2006)$. GQD species and dose used varied between studies. Table 1 summarizes the characteristics of each trial.

\subsection{Pooled Effects of GQD on T2DM}

3.4.1. Improvement of Fasting Plasma Glucose. Twenty-six randomized controlled trials tested the effect of GQD on FPG in patients with T2DM [21-46]. A high level of statistical heterogeneity was observed for the meta-analysis of FBG $\left(I^{2}=99 \%, p<0.00001\right)$, so the random-effect model was used. As presented in Figure 3, the meta-analysis identified a significant decrease of the FPG compared to control group (MD $-1.64,95 \%$ CI $(-2.06,-1.21), p<0.00001)$. Similar results were reported in the subgroup analysis, GQD had a significantly lower FPG than the only metformin group (MD -0.99 , $95 \%$ CI $(-1.63,-0.36), p=0.002$; and GQD plus metformin had a significantly lower FPG than the only metformin group (MD -1.79, 95\% CI $(-2.31,-1.27), p<0.00001)$.

3.4.2. Improvement of 2-Hours Postprandial Plasma Glucose. Twenty-two randomized controlled trials tested the effect of GQD on 2hPPG in patients with T2DM [21-23, 25-28, 30-33, 35-42, 44-46]. A high level of statistical heterogeneity was observed for the meta-analysis of 2hPPG $\left(I^{2}=93 \%, p<0.00001\right)$, so the random-effect model 


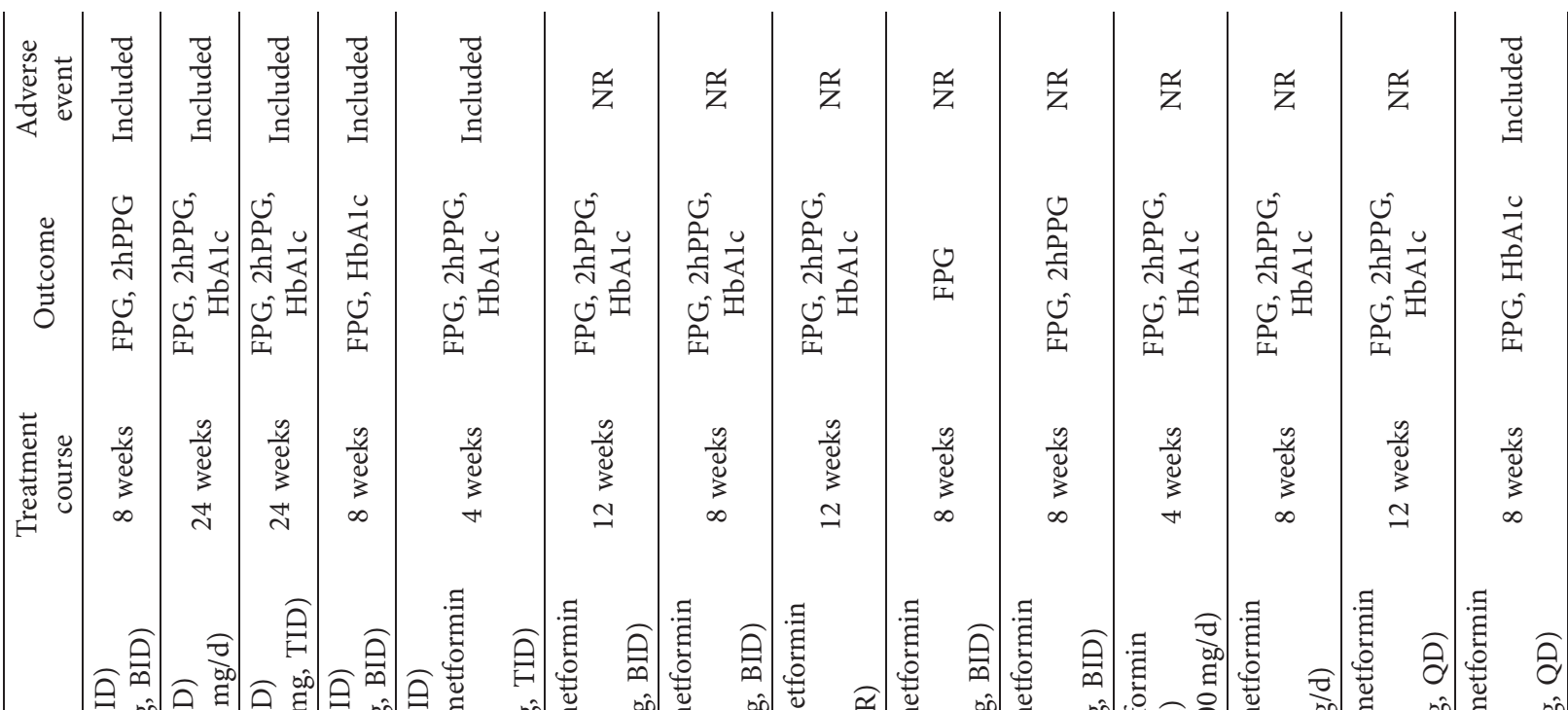

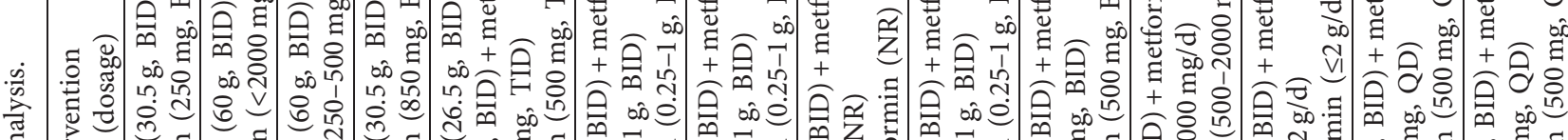

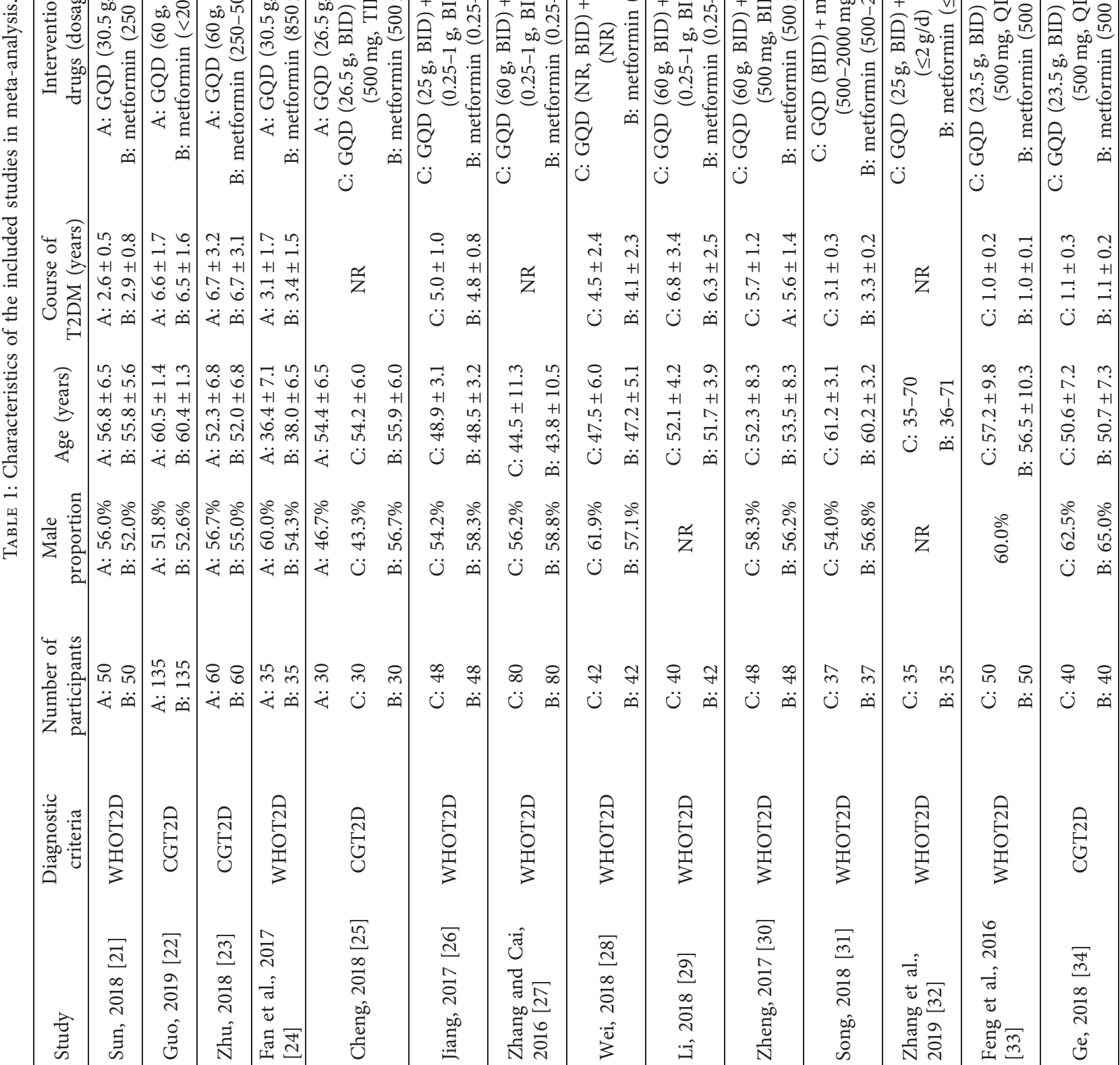









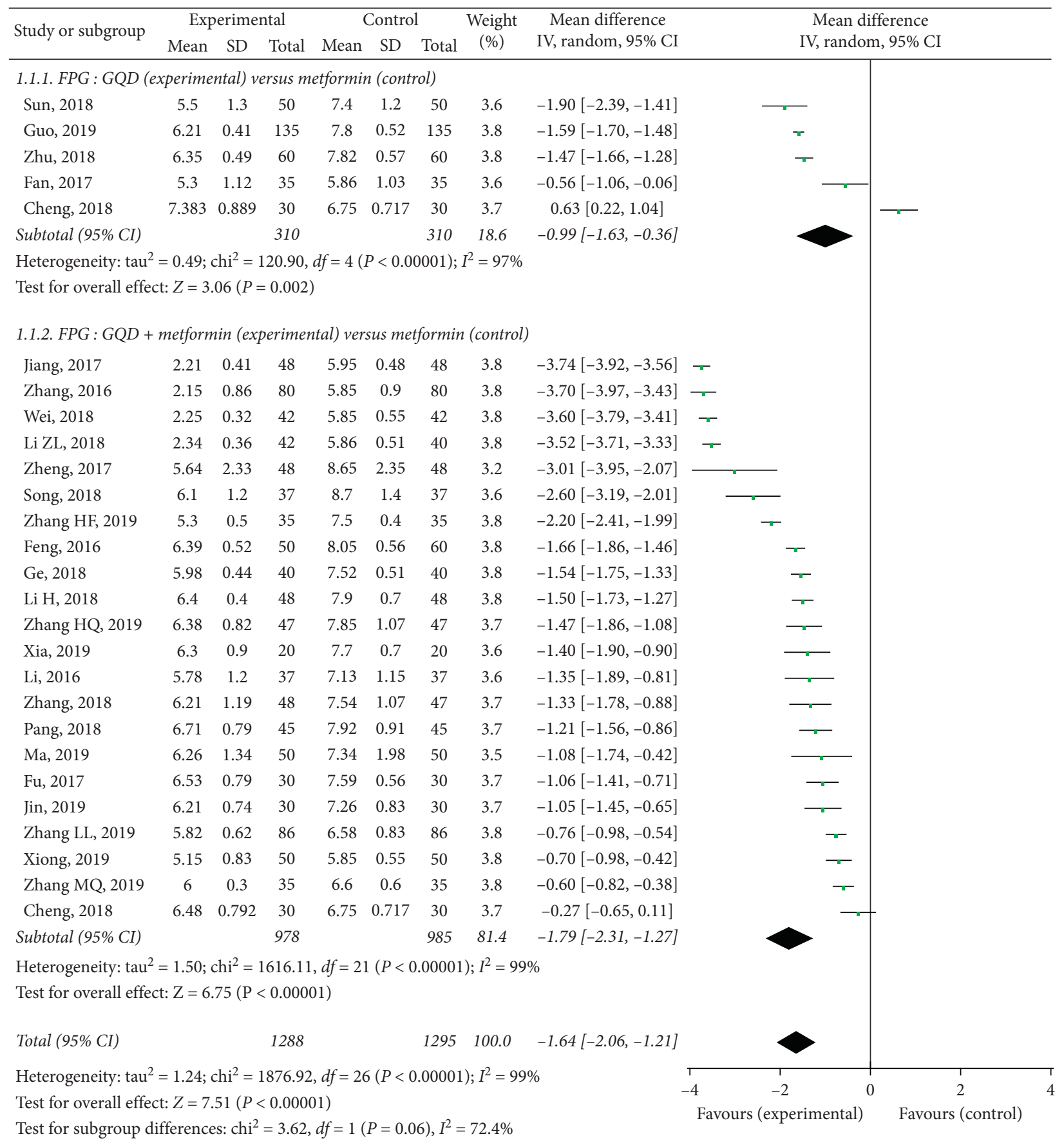

FIGURE 3: Treatment effects of GQD on FPG in patients with type 2 diabetes mellitus. Pooled estimates calculated by the random-effect method. FBG, fasting blood glucose; GQD, Gegen-Qinlian Decoction; CI, confidence interval; and IV, inverse variance.

was used. As presented in Figure 4, results of this metaanalysis showed that GQD could not significantly reduce 2hPPG, as compared to metformin (MD -2.05 , 95\% CI $(-4.15,0.05), p=0.06$. However, GQD plus metformin had a significantly lower $2 \mathrm{hPPG}$ than the only metformin group (MD -1.72, 95\% CI $(-2.12,-1.31), p<0.00001)$.

3.4.3. Improvement of Glycosylated Hemoglobin. Twenty randomized controlled trials tested the effect of GQD on
HbA1c in patients with T2DM [22-28, 31-37, 40, 42-46]. A high level of statistical heterogeneity was observed for the meta-analysis of $\mathrm{HbA1c}\left(I^{2}=99 \%, p<0.00001\right)$, so the random-effect model was used. As presented in Figure 5, results of this meta-analysis showed that GQD could not significantly reduce $\mathrm{HbA} 1 \mathrm{c}$, as compared to metformin (MD $-0.49,95 \%$ CI $(-1.15,0.17), p=0.14)$. However, GQD plus metformin had a significantly lower HbA1c than the only metformin group (MD $-1.26,95 \%$ CI $(-1.80,-0.72)$, $p<0.00001)$. 


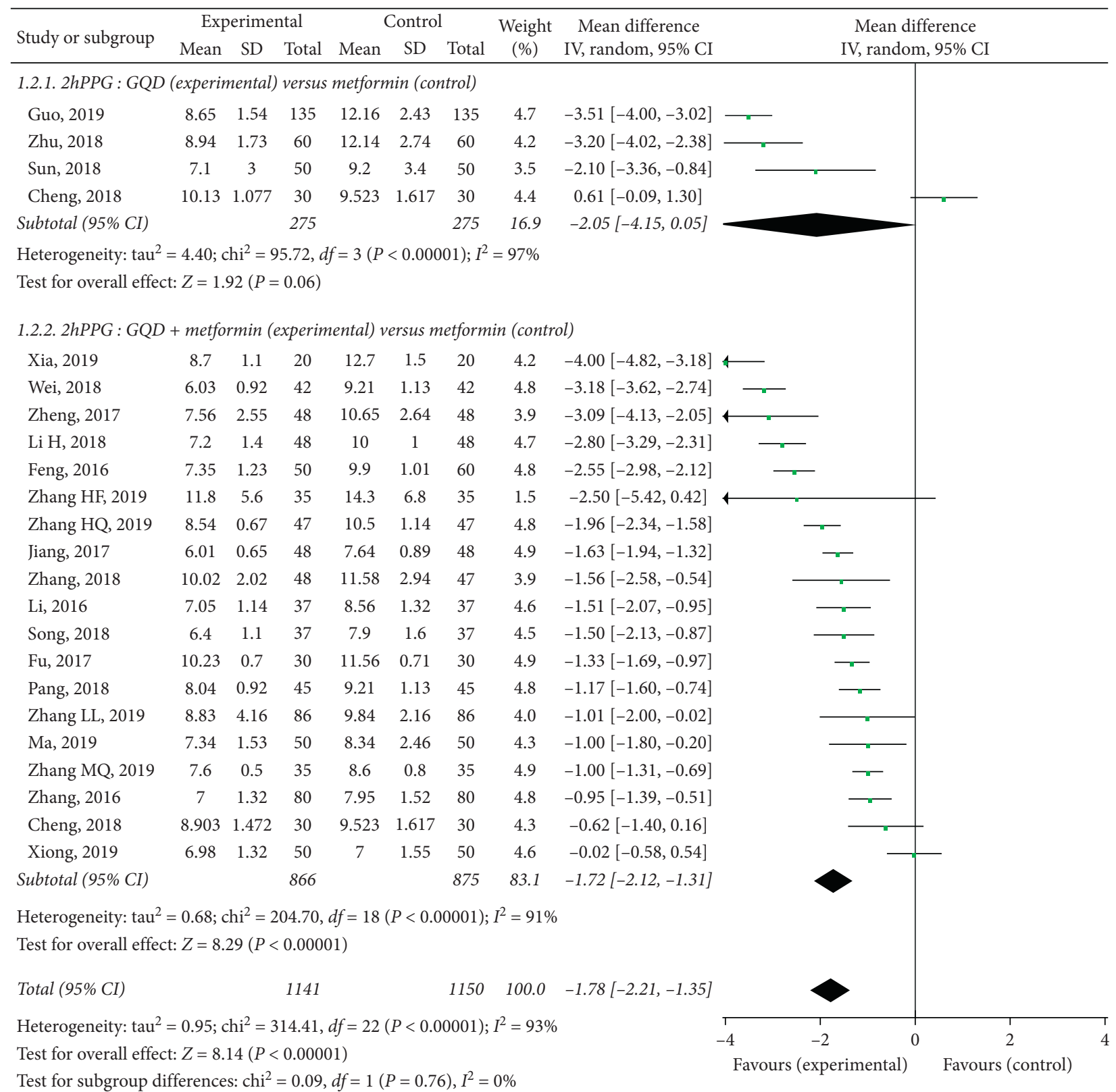

FIGURE 4: Treatment effects of GQD on $2 \mathrm{hPPG}$ in patients with type 2 diabetes mellitus. Pooled estimates calculated by the random-effect method. 2hPPG, 2-hour postprandial plasma glucose; GQD, Gegen-Qinlian Decoction; CI, confidence interval; and IV, inverse variance.

3.5. Publication Bias. The prevention of publication bias is important for the scientific perspective. In this study, the funnel plots showed that no evidence of publication bias was apparent in the 26 clinical trials Figure 6, and Egger's test also indicated no significant publication bias $(p=0.2470)$.

\section{Adverse Events}

Eleven RCTs reported information on adverse effects [21-25, 34, 36, 41, 43, 44, 46]. There were no serious adverse reactions in the RCTs, mostly mild to moderate gastrointestinal reactions. In these RCTs, the most common adverse events were nausea, vomiting, diarrhea, headache, and hypoglycemia. The adverse events of GQD were nausea, vomiting, diarrhea, and hypoglycemia, which is similar to the side effects of metformin $[23,24]$. However, the adverse events in the combination of GQD and metformin were decreased significantly in three studies [41, 43, 46], as compared to the metformin group.

\section{Discussion}

In total, this study assessed the efficacy and safety of GQD in adult patients with T2DM. Review Manager 5.4 software was used to analyze the clinical data from 26 RCTs, with a total of 2553 participants. All trials were carried out in China, and all the patients involved were Chinese. The results showed that the combination of GQD and metformin was more effective 


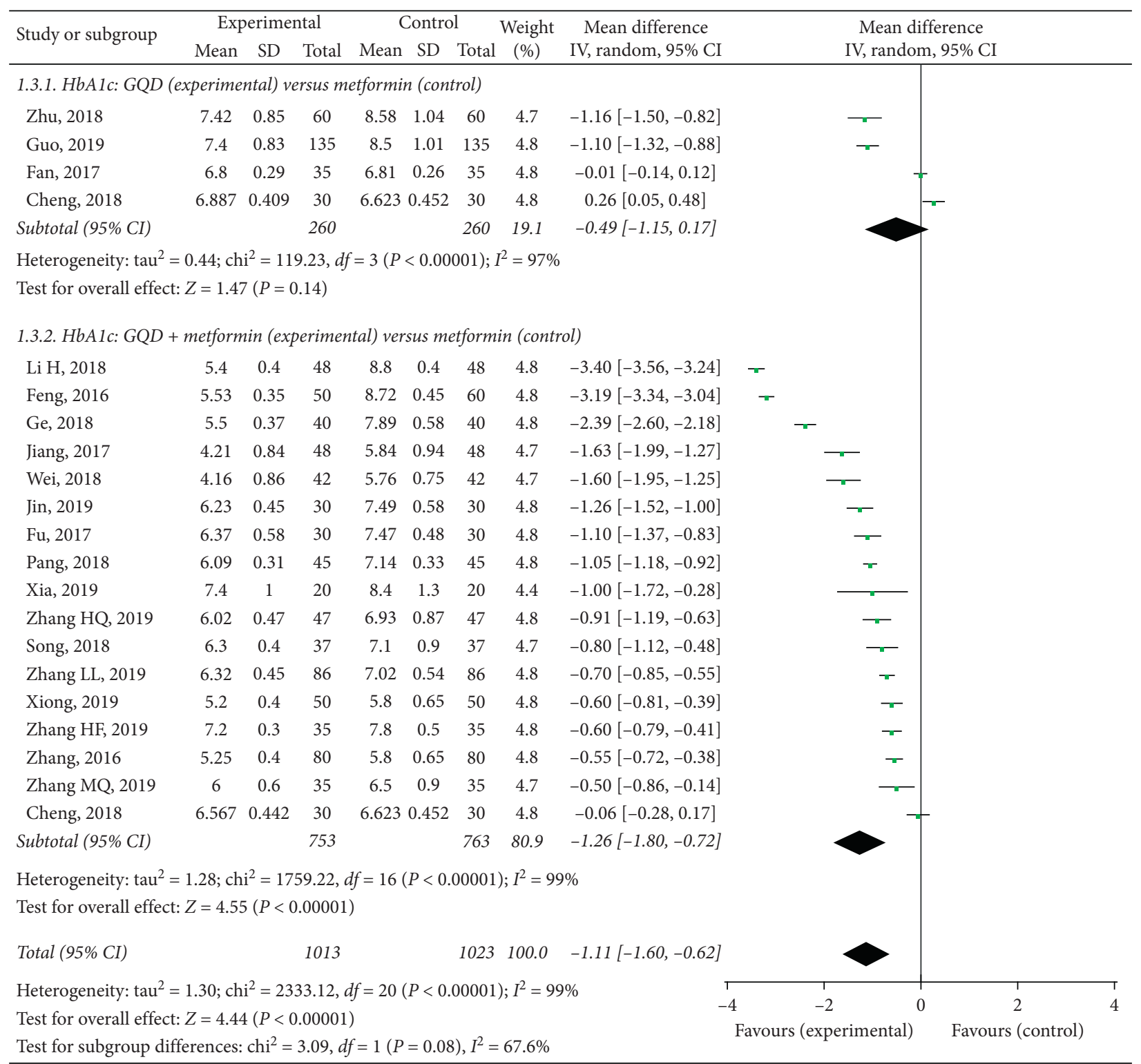

FIgURE 5: Treatment effects of GQD on HbA1c in patients with type 2 diabetes mellitus. Pooled estimates calculated by the random-effect method. HbA1c, Glycosylated hemoglobin; GQD, Gegen-Qinlian Decoction; CI, confidence interval; and IV, inverse variance.

in the treatment of T2DM when compared to metformin alone. This study also suggested that GQD was a safe drug for T2DM patients.

Diabetes is an increasingly important condition in the world. In 2011, there are 366 million patients with diabetes, and it is expected to rise to 552 million by 2030 [47]. T2DM is part of a complex metabolic-cardiovascular syndrome, and metformin is recommended as first-line oral therapy in most national and international guidelines. However, a considerable number of patients need to add other drugs on the basis of first-line metformin over time [48]. Traditional Chinese medicine advocates the use of multiple herbal medicines in combination, which can not only produce multiple effects but also reduce adverse reactions [49]. In the recent years, GQD has played an important role in the treatment of T2DM in China [9, 10]. Many studies have found that the chemical components of GQD are related to the pathological factors of T2DM. Puerarin, the component of Puerariae Lobatae Radix, plays a role in reducing blood sugar by promoting insulin expression and improving glucose metabolism [16]. The flavonoids of Puerariae Lobatae Radix not only have a significant hypoglycemic effect but also can prevent the diabetic complications [50]. Berberine, the component of Coptidis Rhizoma, has significant effects on reducing blood glucose and blood lipid, improving insulin resistance and egulating intestinal tract flora [51]. Baicalin, the main active ingredient of Scutellariae Radix, can promote glucose uptake and glycolysis, inhibit gluconeogenesis, and improve glucose metabolism [52]. Isoliquiritigenin and liquiritigenin, the components of 


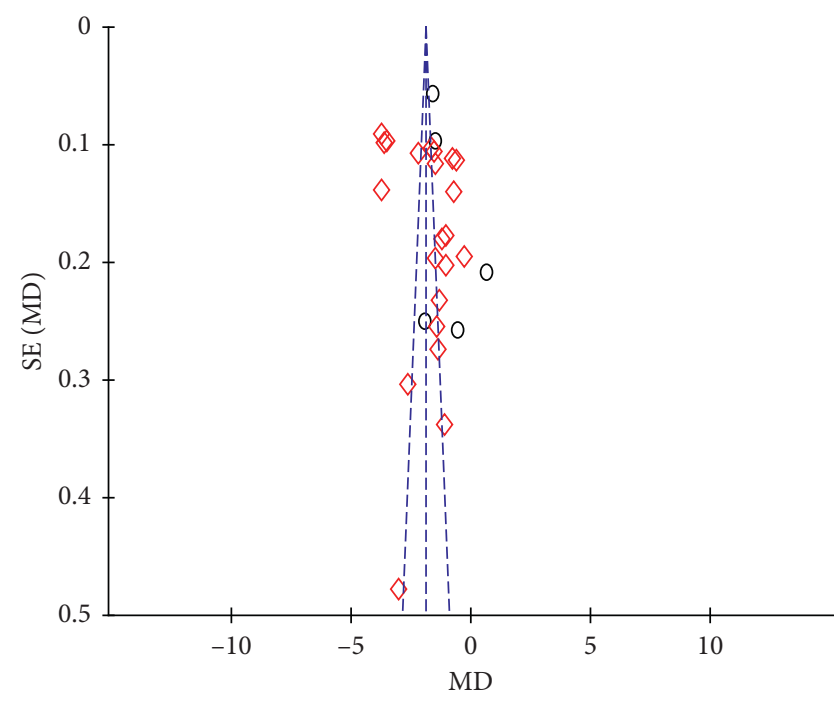

$$
\begin{aligned}
& \text { Subgroups } \\
& \text { O FPG : GQD (experimental) versus metformin (control) } \\
& \diamond \text { FPG : GQD + metformin (experimental) versus } \\
& \text { metformin (control) }
\end{aligned}
$$

FIGURE 6: Funnel plots of randomized controlled trials of GQD. GQD, Gegen-Qinlian Decoction; FBG, fasting blood glucose.

Glycyrrhizae Radix et Rhizoma, can reduce insulin resistance in liver [53]. It is noteworthy that the active ingredients of GQD have produced a potential effect of multitarget therapy. The explanation might be that a disease, such as T2DM, with most likely a number of various mechanisms involved, is likely to respond to a multitarget treatment.

Metformin can reduce glucose production and partially increased glucose utilization, while GQD can accelerate the absorption and utilization $[5,9,10,48,50-54]$. These findings suggest that the combined use of GQD and metformin may be more effective than metformin alone. Indeed, as shown in Figures 3-5, the present study included 26 RCTs involving 2553 patients. The results showed that compared with metformin, GQD plus metformin significantly improve FPG (MD - 1.64, $p<0.00001$ ), 2hPPG (MD $-1.78, p<0.00001)$, and also significantly improve the HbAlc (MD -1.11, $p<0.00001$ ). Additionally, the GQD plus metformin showed a significant reduction in adverse events when compared with the patients without GQD treatment.

In 2017, the study of Ryuk et al. [7] confirmed the synergistic effect of GQD plus metformin on glucose control, which included 5 RCTs with a total of 499 participants. In our study, which is based on the other three indicators (FPG, 2hPPG, and $\mathrm{HbAlc}$ ), we further determine the effectiveness and safety of GQD in the treatment of T2DM. FPG is an indicator for the diagnosis of diabetes. $2 \mathrm{hPPG}$ provides more information on postprandial glycemic control and is important in prognostic indicators such as cardiovascular disease, renal failure, or diabetic amputation. HbAlc provides information on overtime blood glucose control [55]. In addition, more RCTs (26 studies included) and more participants (more than 2500 patients) were included in this study, and the results further confirmed that the combination of GQD and metformin was more effective compared to metformin alone in the treatment of T2DM with no serious side effects.

The present meta-analysis has several potential limitations that should be addressed. First, the therapeutic effect of herbal formula has been gradually recognized by international medical community. But, all RCTs included are conducted in China and published in Chinese, which has seriously affected the international communication of GQD. Secondly, traditional Chinese medicine has always emphasized individualized treatment based on clinical symptoms, so different medicines, different doses, and different courses of treatment may lead to heterogeneity. The high heterogeneity is observed among included RCTs, which will influence the analysis, interpretation, and conclusions of this study. Third, quality control of herbal formula has been necessary and urgent for the safety and efficacy of GQD, but all the RCTs lack sufficient information on the quality control. Fourth, the traditional decoction is influenced by many factors, such as the quality of medicinal materials and the method of decocting and taking. In the recent years, some new forms of herbal formula (such as capsules or tablets) have been studied and popularized, which helps GDQ to be safer and more stable in quality and curative effect $[56,57]$. Finally, as shown in Figure 2, lacking of detailed demographic and methodological information in many studies (such as medication history, sequence generation, and dropout rates) leads to the poor methodological quality. Despite the limitations, this study confirms that GQD is indeed a safe and effective adjunct to metformin for the treatment of T2DM, and the consistent and highly significant are very compelling.

\section{Conclusions}

This study could not provide adequate evidence to conclude whether GQD is superior, inferior, or the same as metformin in terms of efficacy for the treatment of T2DM. However, the hypoglycemic effect of metformin is significantly enhanced when it is combined with GQD, and no serious side effects are identified. Due to overall limited quality of included studies, the therapeutic benefit of GQD can be substantiated to a limited degree. Future studies are needed to address the effectiveness and safety of GQD with larger sample size and better methodological quality across diverse populations.

\section{Data Availability}

Data that support the results of this study are included within the article.

\section{Conflicts of Interest}

All authors declare that they have no conflicts of interest. 


\section{Acknowledgments}

This work was supported by the Health Commission of Sichuan Province (20PJ063) and the Chengdu Science and Technology Program (2019-YFYF-00087-SN).

\section{References}

[1] American Diabetes Association, "2. classification and diagnosis of diabetes: standards of medical care in diabetes-2019," Diabetes Care, vol. 42, no. Suppl 1, p. S13-S28, 2019.

[2] S. Chatterjee, K. Khunti, and M. J. Davies, "Type 2 diabetes," The Lancet, vol. 389, no. 10085, p. 2239, 2017.

[3] A. Trikkalinou, A. K. Papazafiropoulou, and A. Melidonis, "Type 2 diabetes and quality of life," World Journal of Diabetes, vol. 8, no. 4, p. 120, 2017.

[4] J. Ren, F. Ma, Y. Zhou et al., "Hearing impairment in type 2 diabetics and patients with early diabetic nephropathy," Journal of Diabetes and Its Complications, vol. 32, no. 6, p. 575, 2018.

[5] E. Sanchez-Rangel and S. E. Inzucchi, "Metformin: clinical use in type 2 diabetes," Diabetologia, vol. 60, no. 9, p. 1586, 2017.

[6] C. S. Liu, X. Liang, X. H. Wei et al., "Gegen Qinlian decoction treats diarrhea in piglets by modulating gut microbiota and short-chain fatty acids," Frontiers in Microbiology, vol. 10, p. 825, 2019.

[7] J. A. Ryuk, M. Lixia, S. Cao, B.-S. Ko, and S. Park, "Efficacy and safety of Gegen Qinlian decoction for normalizing hyperglycemia in diabetic patients: a systematic review and meta-analysis of randomized clinical trials," Complementary Therapies in Medicine, vol. 33, p. 6, 2017.

[8] H. Li, L. Zhao, B. Zhang et al., "A network pharmacology approach to determine active compounds and action mechanisms of Ge-Gen-Qin-Lian decoction for treatment of type 2 diabetes," Evidence-based Complementary and Alternative Medicine, vol. 2014, Article ID 495840, 12 pages, 2014.

[9] C. H. Zhang, G. L. Xu, Y. H. Liu et al., "Anti-diabetic activities of Gegen Qinlian decoction in high-fat diet combined with streptozotocin-induced diabetic rats and in 3T3-L1 adipocytes," Phytomedicine, vol. 20, no. 3-4, pp. 221-229, 2013.

[10] J. Han, Z. Wang, W. Xing et al., "Effect of Gegen Qinlian decoction on cardiac gene expression in diabetic mice," International Journal of Genomics, vol. 2017, Article ID 7421761, 10 pages, 2017.

[11] X. Y. Wang, Z. Jin, X. Ling et al., "Pharmacokinetic characteristics of fourteen main components of Gegen Qinlian decoction in rats," China Journal of Chinese Materia Medica, vol. 43, no. 23, pp. 4724-4734, 2018.

[12] Q. Wang, W. Song, X. Qiao et al., "Simultaneous quantification of 50 bioactive compounds of the traditional Chinese medicine formula Gegen-Qinlian decoction using ultra-high performance liquid chromatography coupled with tandem mass spectrometry," Journal of Chromatography A, vol. 1454, p. 15, 2016.

[13] Y. Zhang, J. Yuan, Y. Zhang et al., "LC-MS/MS analysis of Gegen Qinlian decoction and its pharmacokinetics after oral administration to rats," Biomedical Chromatography, vol. 29, no. 4, pp. 485-495, 2015.

[14] Z. Yang, W. Huang, J. Zhang, M. Xie, and X. Wang, "Baicalein improves glucose metabolism in insulin resistant HepG2 cells," European Journal of Pharmacology, vol. 854, pp. 187193, 2019.
[15] Z. Li, Y. N. Geng, J. D. Jiang, and W. J. Kong, “Antioxidant and anti-inflammatory activities of berberine in the treatment of diabetes mellitus," Evidence-Based Complementary and Alternative Medicine, vol. 2014, Article ID 289264, 12 pages, 2014.

[16] K. Wu, T. Liang, X. Duan, L. Xu, K. Zhang, and R. Li, “Antidiabetic effects of puerarin, isolated from Pueraria lobata (willd.), on streptozotocin-diabetogenic mice through promoting insulin expression and ameliorating metabolic function," Food and Chemical Toxicology, vol. 60, pp. 341-347, 2013.

[17] J. Cheel, P. V. Antwerpen, L. Tưmová et al., "Free radicalscavenging, antioxidant and immunostimulating effects of a licorice infusion (Glycyrrhiza glabra L.)," Food Chemistry, vol. 122, no. 3, pp. 508-517, 2010.

[18] Chinese Diabetes Society, "China guideline for type 2 diabetes," Chinese Journal of Endocrinology and Metabolism, vol. 30, no. 10, pp. 26-89, 2014.

[19] World Health Organization Expert Committee, "Definition, diagnosis and classification of diabetes mellitus and its complications. Report of a WHO consultation, part 1: diagnosis and classification of diabetes mellitus," World Health Organization, Geneva, Switzerland, 1999.

[20] H. Gong, F. Qin, and H. He, "Herbal formula modified buzhong-yiqi-tang for functional constipation in adults: a meta-analysis of randomized controlled trials," Evidencebased Complementary and Alternative Medicine, vol. 2018, Article ID 9602525, 12 pages, 2018.

[21] H. Sun, "Analysis of clinical effect of TCM in treatment of diabetes," Diabetes New World, vol. 21, no. 9, pp. 95-96, 2018.

[22] T. T. Guo, "Clinical observation on the treatment of type 2 diabetes by Gegen Qinlian decoction," Diabetes New World, vol. 22, no. 18, pp. 65-66, 2019.

[23] Y. G. Zhu, "Clinical observation on 60 cases of type 2 diabetes treated with Gegen Qinlian decoction," Chinese Journal of Ethnomedicine and Ethnopharmacy, vol. 27, no. 1, pp. 114-115, 2018.

[24] Y. F. Fan, W. Cao, Y. X. Hu et al., "Therapeutic effectiveness of Gegen Qinlian decoction on newly diagnosed type 2 diabetes mellitus with insulin resistance," Modern Journal of Integrated Traditional Chinese and Western Medicine, vol. 26, no. 2, pp. 115-117, 2017.

[25] M. J. Cheng, "The clinical effect of Gegen Qinlian decoction combined with metformin in treatment of type 2 diabetes mellitus with damp-heat syndrome," Hunan University of Traditional Chinese Medicine, Changsha, China, 2018.

[26] X. M. Jiang, "Clinical effect of Gegen Qinlian decoction on type 2 diabetes," Nei Mongol Journal of Traditional Chinese Medicine, vol. 36, no. 18, p. 75, 2017.

[27] Y. Zhang and C. C. Cai, "Gegen Qinlian decoction in treatment of type 2 diabetes," Liaoning Journal of Traditional Chinese Medicine, vol. 43, no. 4, pp. 783-785, 2016.

[28] D. T. Wei, "Clinical efficacy of Gegen Qinlian decoction in the treatment of type 2 diabetes," Health Care Today, vol. 10, pp. 147-148, 2018.

[29] Z. L. Li, "Observation on the therapeutic effect of Gegen Qinlian decoction on type 2 diabetes of damp heat syndrome," Diabetes World, vol. 15, no. 3, p. 50, 2018.

[30] J. F. Zheng, "Curative effect observation on treating type 2 diabetes mellitus of the Shire syndrome with the Gegen Qinlian decoction," Clinical Journal of Chinese Medicine, vol. 9, no. 36, pp. 36-37, 2017.

[31] Y. Y. Song, "Analysis of the clinical effect of Gegen Qinlian Decoction on type 2 diabetes," Health Guide, vol. 15, p. 36, 2018. 
[32] H. F. Zhang, Y. Q. Zhou, and Z. Y. Zhong, "Effect analysis of Gegen Qinlian decoction combined with metformin in the treatment of type 2 diabetes with dampness-heat syndrome," Chinese Community Doctors, vol. 35, no. 5, pp. 128-129, 2019.

[33] X. G. Feng, Y. Z. Yan, Y. P. Zeng et al., "The effect of Gegen Qinlian decoction on intestinal flora in damp-heat syndrome of type 2 diabetes," World Journal of Integrated Traditional and Western Medicine, vol. 11, no. 8, pp. 1110-1112, 2016.

[34] A. L. Ge, "Effect of Gegen Qinlian decoction on insulin resistance in patients with type 2 diabetes mellitus," Journal of North Pharmacy, vol. 15, no. 11, pp. 43-44, 2018.

[35] H. Li, "Clinical observation on 48 cases of type 2 diabetes treated with compound Gegen Qinlian decoction," Hunan Journal of Traditional Chinese Medicine, vol. 34, no. 8, pp. 65-66, 2018.

[36] H. Q. Zhang, "47 cases of type 2 diabetes mellitus with obesity treated with Gegen Qinlian Decoction," Modern Traditional Chinese Medicine, vol. 39, no. 6, pp. 76-79, 2019.

[37] T. Xia, "Clinical effect of Gegen Qinlian decoction combined with metformin in the treatment of 40 patients with type 2 diabetes," Nei Mongol Journal of Traditional Chinese Medicine, vol. 38, no. 3, pp. 56-57, 2019.

[38] S. B. Li, "Treatment of diabetic patients with Puerariae and Scutellariae and Coptidis decoction effect analysis," Diabetes New World, vol. 19, no. 5, pp. 31-33, 2016.

[39] J. Zhang, Q. Ma, and R. Chen, "Clinical effect and mechanism of Gegen Qinlian decoction on type 2 diabetes mellitus with lower extremity vascular disease," Shaanxi Journal of Traditional Chinese Medicine, vol. 39, no. 1, pp. 86-88, 2018.

[40] X. Y. Pang, X. M. Pang, Y. Li et al., "Clinical study on gegen qinlian decoction for the treatment of type 2 diabetes," $D i$ abetes New World, vol. 21, no. 17, pp. 103-104, 2018.

[41] J. B. Ma, "Clinical observation of Gegen Qinlian decoction in the treatment of diabetes mellitus," Health Guide, vol. 3, p. 237, 2019.

[42] G. Z. Fu, "Gegen Qinlian decoction in patients with obese type 2 diabetes mellitus (damp heat type)," Henan University of Traditional Chinese Medicine, Zhengzhou, China, 2017.

[43] C. Jin, Y. Zhang, R. H. Li et al., "Effect of Gegen Qinlian decoction on glucose and lipid metabolism index and body weight in patients with type 2 diabetes mellitus," Medical Information, vol. 32, no. 14, pp. 164-165, 2019.

[44] L. L. Zhang, L. Yang, and Z. C. Wei, "Clinical evaluation of Gegen Qinlian decoction in the treatment of type 2 diabetes mellitus," Contemporary Medicine, vol. 25, no. 32, pp. 10-12, 2019.

[45] Q. J. Xiong, "Clinical effect of Gegen Qinlian decoction in the treatment of type 2 diabetes," Clinical Research and Practice, vol. 4, no. 26, pp. 148-149, 2019.

[46] M. Q. Zhang, "Analysis of the effect of Gegen Qinlian decoction on diabetic patients," Guide of China Medicine, vol. 17, no. 34, pp. 179-180, 2019.

[47] D. R. Whiting, L. Guariguata, C. Weil, and J. Shaw, "IDF diabetes atlas: global estimates of the prevalence of diabetes for 2011 and 2030," Diabetes Research and Clinical Practice, vol. 94, no. 3, p. 311, 2011.

[48] S. M. Kasper, K. Pernille, K. A. K. Lise et al., "Metformin and second- or third-generation sulphonylurea combination therapy for adults with type 2 diabetes mellitus," Cochrane Database of Systematic Reviews, vol. 4, no. 4, Article ID CD012368, 2019.

[49] F. Qin, X.-A. Wu, Y. Tang, Q. Huang, Z.-J. Zhang, and J.-H. Yuan, "Meta-analysis of randomized controlled trials to assess the effectiveness and safety of free and easy wanderer plus, a polyherbal preparation for depressive disorders," Journal of Psychiatric Research, vol. 45, no. 11, pp. 1518-1524, 2011.

[50] Z. C. Zhang, X. Y. Ye, M. H. Xu, and Y.-f. Wang, "Experimental study on the hypoglycemic action and prevention of diabetic complication with Pueraria flavonoids," Journal of East China Normal University (Natural Science), vol. 2, pp. 77-81, 2010.

[51] C. Li, J. Z. He, X. D. Zhou, and X. Xu, "Berberine regulates type 2 diabetes mellitus related with insulin resistance," Zhongguo Zhong Yao Za Zhi, vol. 42, no. 12, pp. 2254-2260, 2017.

[52] U. Wu, Y. Cha, X. Huang et al., "Protective effects of berberine on high fat-induced kidney damage by increasing serum adiponectin and promoting insulin sensitivity," International Journal of Clinical and Experimental Pathology, vol. 8, no. 11, pp. 14486-14492, 2015.

[53] Y. M. Yang, S. Y. Seo, T. H. Kim, and S. G. Kim, "Decrease of microRNA-122 causes hepatic insulin resistance by inducing protein tyrosine phosphatase $1 \mathrm{~B}$, which is reversed by licorice flavonoid," Hepatology, vol. 56, no. 6, pp. 2209-2220, 2012.

[54] Q. Xue, W. Qi, W. Shuang et al., "A 42-markers pharmacokinetic study reveals interactions of berberine and glycyrrhizic acid in the anti-diabetic Chinese medicine formula gegen-qinlian decoction," Frontiers in Pharmacology, vol. 9, p. $622,2018$.

[55] A. K. Mandal, "In treating diabetes, what is important? Glucose levels or outcome measures?" World Journal of Diabetes, vol. 6, no. 13, p. 1243, 2015.

[56] L. H. Chen, J. N. Liu, Q. Wang, and J. H. Chen, "Fingerprint comparison between gegen qinlian preparations of three different pharmaceutical forms including decoction, dispensing granule and pill," Chromatographia, vol. 69, no. 1-2, pp. 123-127, 2009.

[57] H. Li, Y. Li, J. Tian et al., "Comparative study on HPLC fingerprint of gegen qinlian formula granules and its traditional decoction," World Chinese Medicine, vol. 13, no. 9, pp. 2304-2307, 2018. 